Pacific

Journal of

Mathematics

\title{
MINIMAL TRIPLE POINT NUMBERS OF SOME NON-ORIENTABLE SURFACE-LINKS
}

Shin SATOH 


\title{
MINIMAL TRIPLE POINT NUMBERS OF SOME NON-ORIENTABLE SURFACE-LINKS
}

\author{
SHIN SATOH
}

\begin{abstract}
An embedded surface in $R^{4}$ is projected into $R^{3}$ with the double point set which includes a finite number of triple points. We consider the minimal number of such triple points among all projections of embedded surfaces which are ambient isotopic to a given surface and show that for any non-negative integer $N$ there exists a 2-component non-orientable surface in $R^{4}$ whose minimal triple point number is equal to $2 N$.
\end{abstract}

\section{Introduction.}

In this paper we denote the 4-dimensional Euclidian space by

$$
\mathbf{R}^{4}=\{(x, y, z, w) \mid x, y, z, w \in \mathbf{R}\} .
$$

A surface-link is a 2-dimensional manifold $F$ embedded in $\mathbf{R}^{4}$ locally flatly, each component of which is homeomorphic to a closed surface. In particular, it is called a surface-knot when $F$ is connected, and it is called a 2-knot (resp. a $\mathbf{P}^{2}$-knot) when $F$ is homeomorphic to a 2 -sphere (resp. a projective plane). Two surface-links $F$ and $F^{\prime}$ are equivalent if there exists an orientation preserving homeomorphism of $\mathbf{R}^{4}$ which maps $F$ onto $F^{\prime}$. If $F$ and $F^{\prime}$ are equivalent, we use the notation $F \cong F^{\prime}$. For a surface-link $F$, the type of $F$ is the collection of all surface-links each member in which is equivalent to $F$.

To describe a surface-link, we use the projection image in $\mathbf{R}^{3}$. For convenience, we may assume that the projection $\pi: \mathbf{R}^{4} \longrightarrow \mathbf{R}^{3}$ determined by the $w$-axis is a generic projection for a surface-link $F$; that is, its double point set consists of isolated branch points, double point curves, and isolated triple points. The broken surface diagram or simply the diagram of a surface-link $F$ is the generic projection image $\pi(F)$ such that the upper sheet and the lower sheet along each double point curve are distinguished. (To distinguish upper and lower, we often depict the diagram by erasing a small neighborhood of the curve in the lower sheet.)

Let $D_{F}$ be the diagram of a surface-link $F$. We denote the number of the triple points on $D_{F}$ by $t\left(D_{F}\right)$. Then the minimal triple point number of a 
surface-link $F$, denoted by $t(F)$, is the smallest number of the triple points among all the diagrams of surface-links with the same type as $F$;

$$
t(F)=\min \left\{t\left(D_{F^{\prime}}\right) \mid F^{\prime} \cong F\right\} .
$$

This definition is an analogy to that of the 'minimal crossing number' in classical knot theory. It is shown by Kamada that there exists a 2-knot $K$ with $t(K)>N$ for any non-negative integer $N$ (cf. [6]). And also we have $t(F) \neq 1$ for any surface-link $F$ (cf. [7]). However, the minimal triple point number in 2-knot theory differs from the minimal crossing number in classical knot theory: For instance, $t(F)=0$ does not imply that $F$ is trivial. For example, a 2-knot $K$ is ribbon if and only if $t(K)=0$ (cf. [10]). The purpose of this paper is to prove:

Theorem 1.1. For any positive integer $N$, there exists a 2-component surface-link $F=F_{1} \cup F_{2}$ such that

(i) each $F_{i}$ is a non-orientable surface-knot,

(ii) $\chi\left(F_{i}\right)=2-N(i=1,2)$,

(iii) $e\left(F_{1}\right)=2 N$ and $e\left(F_{2}\right)=-2 N$,

(iv) $\pi_{1}\left(\mathbf{R}^{4}-F\right) \cong\langle a, b \mid a b a=b, b a b=a\rangle$, and

(v) $t(F)=2 N$,

where $\chi$ denotes the Euler characteristic, and e denotes the normal Euler number.

\section{Preliminaries.}

We review some definitions and results on diagrams of surface-links. Refer to [3] for more details.

Let $F$ be a surface-link and $D_{F}$ the (broken surface) diagram of $F$. A sign of a branch point on $D_{F}$ is defined as follows: There are two types of crossing information near a branch point — one is positive (with +1$)$ and the other is negative (with -1) - depicted in Figure 1.

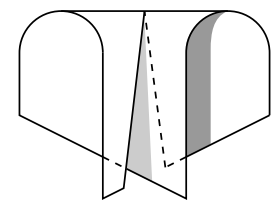

positive

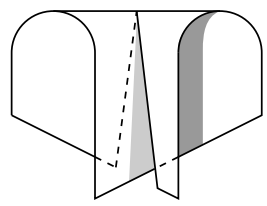

negative

Figure 1.

Proposition $2.1([\mathbf{1}])$. For a surface-knot $F$, the sum of signs taken over all the branch points on $D_{F}$ is equal to the normal Euler number $e(F)$. 
Similarly to the minimal triple point number, we can also consider the minimal branch point number $b(F)$ of a surface-link $F$ as follows;

$$
b(F)=\min \left\{b\left(D_{F^{\prime}}\right) \mid F^{\prime} \cong F\right\},
$$

where $b\left(D_{F^{\prime}}\right)$ is the number of the branch points on the diagram $D_{F^{\prime}}$. In [2], Carter and Saito determined the number $b(F)$ completely as follows (they proves only the case of surface-knots, but their technique used in their paper is also applied for any surface-links).

Proposition $2.2([2])$. For a surface-link $F=F_{1} \cup \cdots \cup F_{n}$, we have

$$
b(F)=\left|e\left(F_{1}\right)\right|+\cdots+\left|e\left(F_{n}\right)\right| .
$$

Let $\Gamma_{F}$ be the double point set of the diagram $D_{F}$, which is regarded as a union of immersed loops and immersed arcs in $\mathbf{R}^{3}$ such that the endpoints of the immersed arcs are branch points.

Suppose that $\Gamma_{F}$ contains a simple arc (that is, an embedded arc with no triple point on it). Such a simple arc is called an a-arc (resp. an $m$ arc) if the two branch points of its ends have the opposite signs (resp. the same sign). We notice that the neighborhood of an $a$-arc (resp. an $m$-arc) is homeomorphic to an annulus (resp. a Möbius band). By canceling the branch points on an $a$-arc as illustrated in Figure 2, we have the following.

Lemma $2.3([\mathbf{9}])$. If $\Gamma_{F}$ contains an a-arc, then $F$ is equivalent to a surface-link $F^{\prime}$ with $t\left(D_{F^{\prime}}\right)=t\left(D_{F}\right)$ and $b\left(D_{F^{\prime}}\right)=b\left(D_{F}\right)-2$.

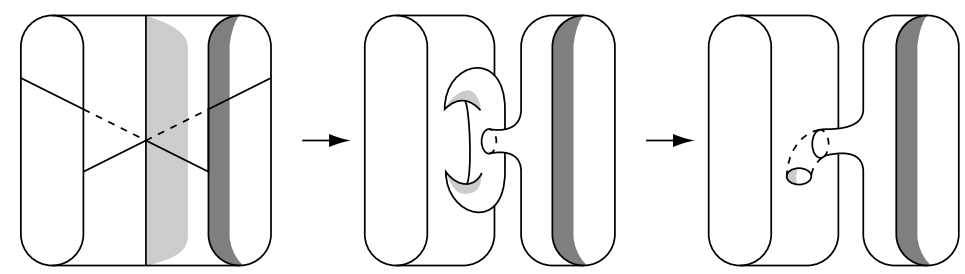

Figure 2.

A surface-link $F$ is $\mathbf{P}^{2}$-reducible if $F$ is equivalent to a connected sum of a standard $\mathbf{P}^{2}$-knot and some surface-link (refer to [5] for a standard $\mathbf{P}^{2}$ knot). $F$ is $\mathbf{P}^{2}$-irreducible if $F$ is not $\mathbf{P}^{2}$-reducible. Since the neighborhood of an $m$-arc is a punctured projective plane properly embedded in a 4-ball as depicted in Figure 3, we have the following.

Lemma 2.4 ([8]). If $\Gamma_{F}$ contains an $m$-arc, then $F$ is $\mathbf{P}^{2}$-reducible. 


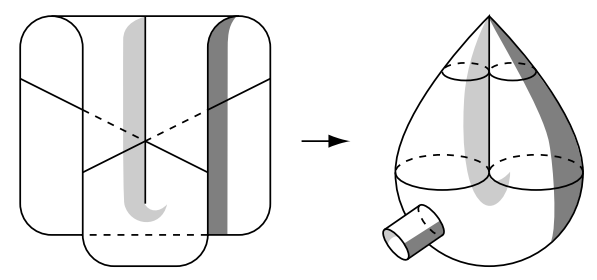

Figure 3.

The neighborhood of a triple point on $D_{F}$ consists of three sheets. These sheets are labeled top, middle and bottom, and these indicate the relative position of the sheets with respect to the $w$-coordinate.

A branch point $b$ and a triple point $t$ on $D_{F}$ are connected by a double point curve $c$ if there exists a simple sub-arc $c$ of $\Gamma_{F}$ whose endpoints are $b$ and $t$. By the deformation of $F$ into $F^{\prime}$ as illustrated in Figure 4, we have the following.

Lemma 2.5 ([8], [11]). Suppose that a branch point $b$ and a triple point $t$ on $D_{F}$ are connected by a double point curve $c$. If the arc $c$ is transverse to the top sheet or the bottom sheet at $t$, then $F$ is equivalent to a surface-link $F^{\prime}$ with $t\left(D_{F^{\prime}}\right)=t\left(D_{F}\right)-1$ and $b\left(D_{F^{\prime}}\right)=b\left(D_{F}\right)$.
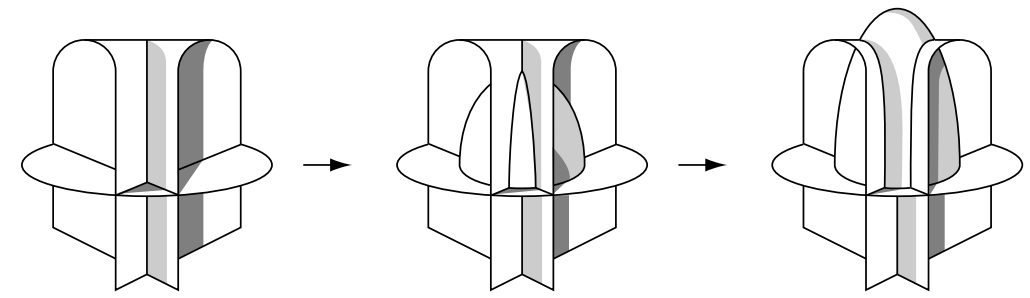

\section{Figure 4.}

Let $\left\{m_{1}, \cdots, m_{n}\right\}$ be a meridian system of a surface-link $F=F_{1} \cup \cdots \cup F_{n}$, where $m_{k}$ is a meridian of $F_{k}(k=1, \cdots, n)$. Each $m_{k}$ is regarded as an element of the knot group $\pi_{1}\left(\mathbf{R}^{4}-F\right)$. Then the following is clear from the property of standard $\mathbf{P}^{2}$-knots.

Lemma 2.6. If the order of each $m_{k}$ is not equal to 2 in $\pi_{1}\left(\mathbf{R}^{4}-F\right)$, then $F$ is $\mathbf{P}^{2}$-irreducible. 


\section{Projections and movie pictures.}

For a $\mathbf{P}^{2}$-irreducible surface-link $F$ we give an estimate for a lower bound of $t(F)$. However, the following lemma has no sense for an orientable surfacelink; for the normal Euler number of any constituent orientable surface-knot vanishes.

Lemma 3.1. For a $\mathbf{P}^{2}$-irreducible surface-link $F=F_{1} \cup \cdots \cup F_{n}$, we have

$$
t(F) \geq\left(\left|e\left(F_{1}\right)\right|+\cdots+\left|e\left(F_{n}\right)\right|\right) / 2,
$$

where $e\left(F_{i}\right)$ denotes the normal Euler number of a surface-knot $F_{i}(i=$ $1, \cdots, n)$.

Proof. By Proposition 2.2, it is sufficient to prove that

$$
t(F) \geq b(F) / 2
$$

for any $\mathbf{P}^{2}$-irreducible surface-link $F$. Let $M$ be the set of all diagrams of the surface-links with the same type as $F$ whose triple point number is realizing $t(F)$

$$
M=\left\{D_{F^{\prime}} \mid F^{\prime} \cong F, t\left(D_{F^{\prime}}\right)=t(F)\right\} .
$$

Among the diagrams in $M$, we take a diagram, say $D$, whose branch point number is minimal in $M$. Let $\Gamma$ be the double point set of $D$.

Since $F$ is $\mathbf{P}^{2}$-irreducible, $\Gamma$ contains no $m$-arc by Lemma 2.4. Moreover $\Gamma$ contains no $a$-arc by Lemma 2.3 ; for if $\Gamma$ contains an $a$-arc, then there exists a diagram $D^{\prime}$ in $M$ with $b(D)>b\left(D^{\prime}\right)$. Hence any branch point in $\Gamma$ is connected with some triple point.

On the other hand, the number of branch points connecting with each triple point in $\Gamma$ is at most two; for if at least three branch points connect with a triple point, then we have a cancelling pair of a branch point and the triple point which satisfies the condition of Lemma 2.5, and so there exists a diagram $D_{F^{\prime \prime}}$ with $F^{\prime \prime} \cong F$ and $t\left(D_{F^{\prime \prime}}\right)<t(F)$. Therefore we have

$$
t(F)=t(D) \geq b(D) / 2 \geq b(F) / 2 .
$$

Corollary 3.2. For a $\mathbf{P}^{2}$-irreducible surface-link $F=F_{1} \cup \cdots \cup F_{n}$, if

$$
t(F)=\left(\left|e\left(F_{1}\right)\right|+\cdots+\left|e\left(F_{n}\right)\right|\right) / 2,
$$

then the minimal triple point number $t(F)$ is even.

Proof. From the proof of Lemma 3.1, there exists a surface-link $F^{\prime} \cong F$ whose diagram $D_{F^{\prime}}$ satisfies $t\left(D_{F^{\prime}}\right)=t(F)$ and $b\left(D_{F^{\prime}}\right)=b(F)$. Let $\Gamma_{F^{\prime}}$ be the double point set of $D_{F^{\prime}}$. Then the neighborhood of each triple point in $\Gamma_{F^{\prime}}$ is as shown in Figure 5(A) or (B). Here the arrows along the double point curves mean a BW orientation of $\Gamma_{F^{\prime}}$ (refer to [7] for a $B W$ orientation of a double point set). Since the number of the triple points depicted in Figure $5(\mathrm{~A})$ is equal to that depicted in Figure $5(\mathrm{~B})$, the sum $t\left(D_{F^{\prime}}\right)$ is even. 


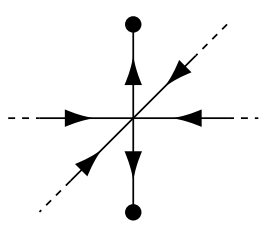

(A)

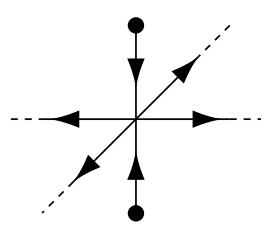

(B)

\section{Figure 5.}

To describe a surface-link $F$, we also use a movie picture method [4]; for any subset $S$ of $\mathbf{R}$, we denote $S \times \mathbf{R}^{3} \subset \mathbf{R} \times \mathbf{R}^{3} \cong \mathbf{R}^{4}$ by $\mathbf{R}^{3} S$. If $S=\left\{x_{0}\right\}$, we write $\mathbf{R}^{3}\left[x_{0}\right]$. Taking the $x$-coordinate as a height function, we consider a surface-link $F$ to be a one-parameter family of subsets in $\mathbf{R}^{3}$ that are the intersections $F_{x}=F \cap \mathbf{R}^{3}[x](-\infty<x<\infty)$. If $F_{x}$ is a classical link, it is called a cross-sectional link.

We consider the relationship between a surface-link described by the projection method and that described by the movie pictured method. Let $\pi^{\prime}$ be the projection $\pi^{\prime}: \mathbf{R}^{3} \longrightarrow \mathbf{R}^{2}$ with $(y, z, w) \longmapsto(y, z)$. Then the projection $\pi: \mathbf{R}^{4} \longrightarrow \mathbf{R}^{3}$ determined by the $w$-axis is considered to be

$$
\text { id } \times \pi^{\prime}: \mathbf{R}^{4} \cong \mathbf{R} \times \mathbf{R}^{3} \longrightarrow \mathbf{R} \times \mathbf{R}^{2} \cong \mathbf{R}^{3} \text {. }
$$

Hence, the projection image $\pi(F)$ of a surface-link $F$ is also considered to be a family of the projection images $\pi^{\prime}\left(F_{x}\right)(-\infty<x<\infty)$.

We notice that a crossing in the (classical link) diagram of each crosssectional link $F_{x}$ corresponds to a double point in the diagram $D_{F}$ of $F$. If consecutive cross-sectional links $\left\{F_{x}\right\}\left(x_{0} \leq x \leq x_{1}\right)$ represent a deformation of a Reidemeister move I (resp. a Reidemeister move III), it produces a branch point (resp. a triple point) in $D_{F}$.

Example 3.3. In Figure 6(A), we depict a 2-component surface $A$ properly embedded in $\mathbf{R}^{3}[0,1]$, each component of which is homeomorphic to a 2 punctured projective plane. Since the projection image determined by the $w$-axis is shown in Figure 6(B), its double point set contains four branch points and two triple points (see Figure $6(\mathrm{C})$ ). 


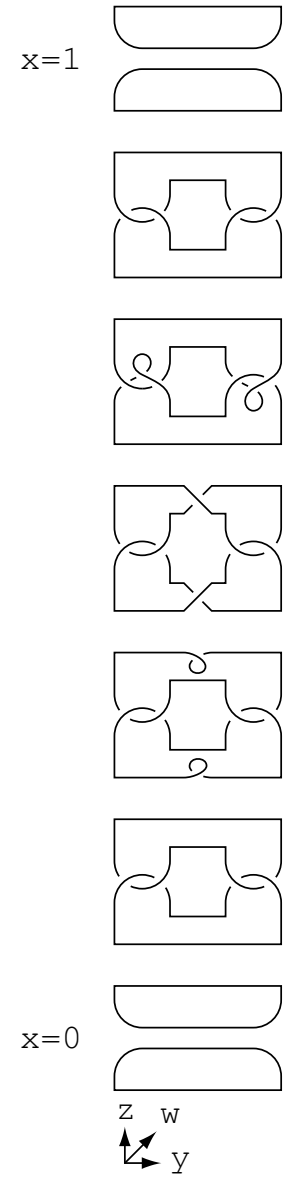

(A)

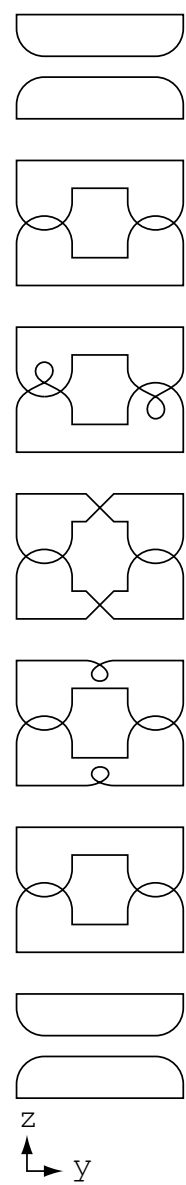

(B)

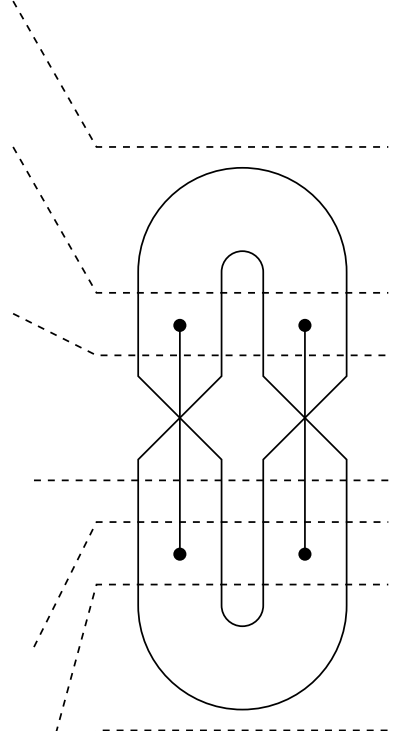

Figure 6.

For any positive integer $N$, we construct a 2-component link $F(N)$ as follows;

$$
F(N) \cap \mathbf{R}^{3}[x]= \begin{cases}B \cup B^{\prime} & \text { for } x=0, \\ A \cap \mathbf{R}^{3}[x] & \text { for } 0<x \leq 1, \\ A \cap \mathbf{R}^{3}[x-1] & \text { for } 1 \leq x \leq 2, \\ \cdots & \\ A \cap \mathbf{R}^{3}[x-(N-2)] & \text { for } N-2 \leq x \leq N-1, \\ A \cap \mathbf{R}^{3}[x-(N-1)] & \text { for } N-1 \leq x<N, \\ B \cup B^{\prime} & \text { for } x=N, \\ \phi & \text { otherwise, }\end{cases}
$$


where $B \cup B^{\prime}$ is a union of two standard 2-disks which bounds the trivial link $A \cap \mathbf{R}^{3}[0]$ ( $=A \cap \mathbf{R}^{3}[1]$ ). Then the double point set of the diagram $D_{F(N)}$ consists of a union of $N$ copies of the set in Figure $6(\mathrm{C})$. We notice that the surface-link $F(1)$ is $8_{1}^{-1,-1}$ in the list of [12].

Proof of Theorem 1.1. We prove that $F(N)$ in Example 3.3 satisfies (i) to (v) in Theorem 1.1. It is easy to verify that each component of $F(N)$ is a (trivial) non-orientable surface-knot with the Euler characteristic $2-N$. The property (iii) is followed by Proposition 2.1 (we recall that a Reidemeister move I corresponds to a branch point). For the calculation of $\pi_{1}\left(\mathbf{R}^{4}-F\right)$, it is useful to refer to [4].

We will only prove that the property $(\mathrm{v}) ; t(F(N))=2 N$. Since the knot group of $F(N)$,

$$
\langle a, b \mid a b a=b, b a b=a\rangle,
$$

is the quaternion group, and since $\{a, b\}$ is a meridian system of $F(N)$, the order of each meridian is 4. By Lemma 2.6, $F(N)$ is $\mathbf{P}^{2}$-irreducible. Hence by the property (iii) and Lemma 3.1, we have $t(F(N)) \geq(|2 N|+$ $|-2 N|) / 2=2 N$. On the other hand, $F(N)$ has the diagram whose double point set contains $2 N$ triple points as shown in Example 3.3. So we have $t(F(N)) \leq 2 N$.

\section{References}

[1] T.F. Banchoff, Double tangency theorems for pairs of submanifolds, in Geometry Symposium Utrecht 1980, ed. Looijenga, Seirsma, and Takens, LNM, Vol. 894, SpringerVerlag, (1981), 26-48.

[2] J.S. Carter and M. Saito, Canceling branch points on projections of surfaces in 4space, Proc. of the A.M.S., 116(1) (Sept. 1992), 229-237.

[3] _ Knotted surfaces and their diagrams, Surveys and monographs, Vol. 55, A.M.S., Jan. 1998.

[4] R.H. Fox, A quick trip through knot theory, in Topology of 3-manifolds and related topics (Georgia, 1961), 120-167, Prentice-Hall.

[5] S. Kamada, Non-orientable surfaces in 4-space, Osaka J. Math., 26 (1989), 367-385.

[6] 2-dimensional braids and chart descriptions, in topics in knot theory (Erzurum/Turkey, 1992), 277-287, NATO ASI Ser. C, 399, Kluwer Academic Publ.

[7] S. Satoh, Lifting a generic surface in 3-space to an embedded surface in 4-space, to appear in Topology Appl.

[8] _ On non-orientable surfaces in 4-space which are projected with at most one triple point, to appear in Proc. of the A.M.S.

[9] _ On simply knotted tori in $S^{4}$ II, in Proceedings of Knots 96 (1997), World Scientific Publishing Co., 551-568.

[10] T. Yajima, On simply knotted spheres in $\mathbf{R}^{4}$, Osaka J. Math., 1 (1969), 133-152. 
[11] S. Yamagata, Some conditions and some examples for maps which are projections of 2-knots, Master thesis, Hiroshima Univ., 1996 (in Japanese).

[12] K. Yoshikawa, An enumeration of surfaces in four space, Osaka J. Math., 31 (1994), 497-522.

Received April 20, 1999 and revised July 7, 1999.

OSAKA UNIVERSITY

Sugimoto, Sumiyoshi-ku, Osaka, 558-5858

JAPAN

E-mail address: susato@sci.osaka-cu.ac.jp 